\title{
Effect of Instrument Navigation on C-arm Radiation and Time during Spinal Procedures: A Clinical Evaluation
}

\author{
TIMOTHY Y. WANG, MD,${ }^{1}$ FARAH HAMOUDA, BS, ${ }^{2}$ VIKRAM A. MEHTA, MD, MPH, ${ }^{1}$ ERIC W. \\ SANKEY, MD, ${ }^{1}$ CHESTER YARBROUGH, MD ${ }^{1}$ ROBERT LARK, MD ${ }^{1}$ MUHAMMAD M. ABD-EL-BARR, \\ $\mathrm{MD}, \mathrm{PHD}^{1}$ \\ ${ }^{I}$ Duke University Medical Center, Department of Neurological Surgery, Durham, North Carolina, ${ }^{2}$ TrackX Technology, LLC, Chapel Hill, North Carolina
}

\begin{abstract}
Introduction: As minimally invasive spine surgery gains popularity, a focused effort must be made to reduce intraoperative radiation exposure to levels as low as reasonably achievable. Here, we demonstrate the clinical efficacy of a novel technology to aid in instrument navigation that aims to reduce intraoperative radiation exposure, number of fluoroscopic images, and time required to perform the most radiation intensive portions of a multitude of spinal procedures.

Methods: An internally randomized controlled study was performed over a 1-month period in order to clinically evaluate the effect of the C-arm assisted instrument tracking system, TrackX, on surgeon workflow, time, and radiation emitted. Three surgeons performed multiple spinal procedures on a total of 10 study patients and an additional 3 control patients. The surgeries encompassed minimally invasive spinal techniques and spanned extreme lateral interbody fusion, oblique lumbar interbody fusion, transforaminal lumbar interbody fusion along with percutaneous iliac screw placement, hardware removal, and kyphoplasty. The tasks studied included skin marking, first dilator insertion, localization for hardware placement and hardware removal.

Results: Overall radiation reduction was $83 \%(P<.0001)$. Overall reduction in x-rays taken was $78 \%(P<.0001)$. Overall time reduction was $81 \%(P=.0003)$. Statistical significance held for each surgeon studied and for nearly every procedure type. In these 10 study procedures, over 2 hours of overall operating room time was saved, all while requiring negligible set up time and no system calibration or supplementary x-rays to be taken. There were no adverse outcomes for any study patient, and there was no case where TrackX was not able to successfully complete a given portion of a procedure.

Conclusions: TrackX instrument navigation is a clinically efficacious and accurate instrument tracking modality. This is the first instrument navigational technology that reduces radiation exposure and images required to complete a procedure while decreasing operative time. TrackX thus allows increased surgical efficiency while increasing operative efficiency and improving intraoperative safety.
\end{abstract}

Level of Evidence: 2.

New Technology

Keywords: instrument tracking, radiation, time, navigation, C-arm, fluoroscopy, spine, image guided surgery, minimally invasive surgery

\section{INTRODUCTION}

Minimally invasive spine surgery (MISS) has a number of clinical benefits, including decreased blood loss, shorter hospitalization, decreased blood loss, and lower infection rates ${ }^{1}$; however, one of the primary drawbacks to MISS is increased radiation exposure to the patient, ${ }^{2}$ surgeon, ${ }^{3,4}$ and operating room (OR) staff. $^{5}$ This is due to the increased reliance on fluoroscopy for visualization of anatomy that would otherwise be exposed during open spine surgery.

This increase in radiation exposure is not without consequence. As MISS continues to mature, new data detailing the detrimental effects of occupational radiation exposure have emerged, suggesting that although minimally invasive approaches benefit patients in the short term, it can have unintended longterm health consequences. These include but are not limited to soft tissue and bloodborne cancers and cataracts. ${ }^{1,3,4}$ Healthcare practitioners, including the surgeon, assistant, scrub nurse, circulator, and anesthesia personnel are arguably at higher risk for developing these unintended consequences, given the repetitive exposure delivered over many cases.

The movement to reduce intraoperative radiation has led to the development of a multitude of new technologies, most of which are aimed at reducing exposure during the instrumented portions of surgical procedures. Many of these technologies 

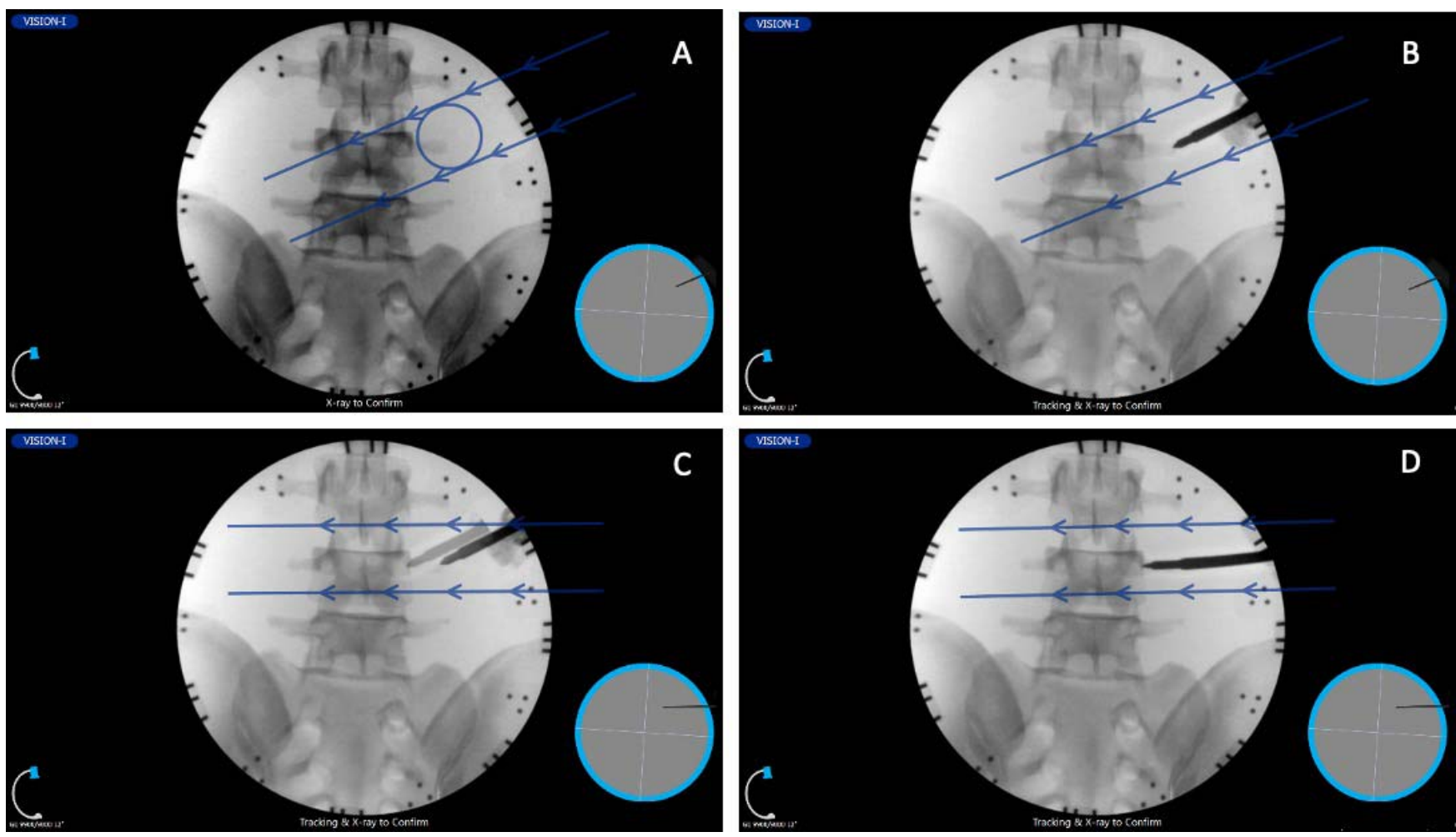

Figure 1. Anterior-posterior visualization of L3-L5 vertebra and sacrum as displayed within the instrument navigation software. (A) X-ray image taken with instrumentation intentionally placed in incorrect location (to demonstrate concept) with approximated instrument tip location (within the circle) and trajectory (direction of the arrows). (B) A subsequent fluoroscopic image demonstrates actual initial instrument placement, which is too lateral to the desired location. (C) Instrument tracking is activated, and thus the instrument can then be traced in virtual live fluoroscopy to the desired location without the use of additional fluoroscopic images. Note the image of the instrument is moving over the anatomy. The moving tip locates the new tip position, and the arrows indicate changed trajectory. (D) Final fluoroscopic image confirms the instrument positioning (3 total fluoroscopic images).

use preoperative or static intraoperative imaging as well as a patient-mounted reference tracker to assist with instrument navigation. Because these systems rely on preoperative imaging gathered weeks to months prior to surgery or static 1-time intraoperative imaging (ie, intraoperative computed tomography [CT]) for instrument navigation, they are unable to account for patient movement or any intraoperative changes in patient anatomy, which would bring the system out of calibration and necessitate another scan to proceed with the case. ${ }^{6-8}$ Although the use of previously described navigation technologies has been shown to decrease the amount of radiation that the practitioners receive, ${ }^{6-8}$ it is associated with a substantial increase in patient radiation and OR time, ${ }^{8}$ as well as disruption of surgeon workflow. ${ }^{9}$

TrackX (TrackX Technology, LLC, Chapel Hill, NC) is a novel instrument-navigation technology meant to overcome these challenges. It functions to use standard intraoperative fluoroscopy to provide instrument navigation (Figure 1). Navigational spheres are placed on a low-profile frame attached to the radiation source and camera of the C-arm fluoroscope. Additional navigational clips are placed on the instrument, which subsequently allows the surgeon to track the instrument in relation to the Carm fluoroscope. After a fluoroscopic image is taken, any movement in the instrumentation will be continuously updated and displayed in real time on the TrackX monitor, which reduces the number of images required to localize and relocalize instrumentation. This also eliminates the need for an invasive reference tracker and any progressive inaccuracy that plagues classic spinal navigation. Although the benefits from time savings and total OR radiation reduction have been shown in cadaveric testing, to date no study in live subjects has been performed. ${ }^{10}$ Accordingly, a multisurgeon, nonblinded, clinical and technical feasibility study in live subjects was designed to compare conventional with intraoperative fluoroscopy with TrackX instrument tracking.

\section{MATERIALS AND METHODS}

A technical feasibility study in live subjects using navigated instrumentation and conventional fluo- 
Table 1. Minimally invasive intervention types studied.

Extreme lateral interbody fusion

Oblique lumbar interbody fusion

Transforaminal lumbar interbody fusion

Percutaneous iliac screw placement

Kyphoplasty

Existing hardware removal

roscopy was performed comparing radiation production, operative time, and number of images required to complete a multitude of individual steps within an array of minimally invasive spine surgeries. Per the institution's request, under a quality initiative protocol exempt from institutional review board approval, surgeons were tasked to perform instrument navigation-assisted spinal procedures on up to 10 study patients. Patients were consented prior to surgery. See Table 1 for the applicable case types studied. Further, any additional patients who had the same type of cases during this time frame performed by the same surgeons were used as additional control data.

For procedures placed in the conventional arm of the study, intraoperative fluoroscopy proceeded without the aid of the instrument tracking system. The study arms were split between 2 paramedian sides in the experimental cases or 1 or more of the levels as applicable. For example, in a 2-level extreme lateral interbody fusion, the each of the 2 initial dilator placements to the disc space would be randomized to the conventional or navigational arm. For portions that were conducted using instrument navigation, navigational snaps as described above were attached to surgical instruments and surgeons were allowed to proceed with the aid of navigation.

Whenever possible, individual portions of the procedure that could be performed twice (ie, pedicle screw insertion or Jamshidi placement) on alternating sides were equally split to either instrument navigated or conventional fluoroscopy. In this fashion, we would minimize confounding factors such as body habitus and individual anatomy that could confound the accuracy of the imaging. The number of x-ray images taken, radiation emitted by the fluoroscope, and time to perform each task within a given procedure were recorded. The method of imaging and navigation were determined preoperatively with a coin flip. During the time of the study, given that this was a limited trial, for cases that did not use the technology (eg, once a given surgeon finished their case allotment for the
Table 2. Studied tasks associated with each case in chronological order.

\begin{tabular}{|c|c|c|c|c|}
\hline Case & $\begin{array}{c}\text { Skin } \\
\text { Marking }\end{array}$ & $\begin{array}{l}\text { First Dilator } \\
\text { Insertion }\end{array}$ & $\begin{array}{l}\text { Hardware } \\
\text { Placement }\end{array}$ & $\begin{array}{c}\text { Hardware } \\
\text { Removal }\end{array}$ \\
\hline 1 & $\mathrm{x}$ & $\mathrm{X}$ & $\mathrm{x}$ & $\mathrm{X}$ \\
\hline 2 & & & $\mathrm{x}$ & \\
\hline 3 & $\mathrm{x}$ & & $\mathrm{x}$ & \\
\hline 4 & $\mathrm{x}$ & & & $\mathrm{x}$ \\
\hline 5 & & & $\mathrm{x}$ & \\
\hline 6 & & & $\mathrm{x}$ & \\
\hline 7 & $\mathrm{x}$ & $\mathrm{x}$ & & \\
\hline 8 & & & & \\
\hline 9 & $\mathrm{x}$ & $\mathrm{x}$ & $\mathrm{x}$ & \\
\hline 10 & $\mathrm{x}$ & $\mathrm{x}$ & $\mathrm{x}$ & \\
\hline 11 & $\mathrm{x}$ & & $\mathrm{x}$ & \\
\hline 12 & & & $\mathrm{x}$ & \\
\hline 13 & $\mathrm{x}$ & & & \\
\hline
\end{tabular}

technology but the study remained open), those same tasks were recorded in these control cases to supplement the control arm data. For each type of case studied in Table 1, these procedures were broken down into common tasks for analysis, where time, x-rays taken, and radiation emitted were recorded for each task within a given procedure. These tasks were as follows: skin marking, first dilator insertion, and localization for hardware placement and hardware removal. The tasks associated with each of the 13 studied cases are listed in Table 2. As such, a 1-level transforaminal lumbar interbody fusion could be studied for up to 6 tasks: skin marking, first dilator insertion, and 4 localizations for Jamshidi needle placement for pedicle screw placement.

\section{Statistical Methods}

Data were collected and analyzed using Excel 2016 (Microsoft, Inc, Redmond, WA), and further statistical analysis was conducted using JMP Pro 13 (SAS Institute, Cary, NC) using a Student $t$ test and 1 -way mean effect analyses with $\alpha<.05$.

\section{RESULTS}

Three surgeons each performed multiple procedures on a total of 10 study patients and an additional 3 control patients. Seventy-eight total tasks were identified, of which 41 tasks could be directly compared with the imaging counterparts; the identical procedure was being performed at multiple levels or an identical procedure was performed contralaterally so that both techniques could be used. All averages were weighted to the number of tasks that corresponded.

Overall, there was a significant decrease in time, number of x-rays, and total radiation when tasks 
Table 3. Comparison of radiation, number of $x$-rays, and operative time between tasks performed with conventional fluoroscopy and TrackX.

\begin{tabular}{|c|c|c|c|c|c|c|c|c|c|c|c|c|}
\hline & \multicolumn{4}{|c|}{ Time, $s$} & \multicolumn{4}{|c|}{ Number of X-Rays } & \multicolumn{4}{|c|}{ Radiation, mGy } \\
\hline & Conventional & TrackX & $\Delta, \%$ & $P$ & Conventional & TrackX & $\Delta, \%$ & $P$ & Conventional & TrackX & $\Delta, \%$ & $P$ \\
\hline Skin marking & $122.5 \pm 118.5$ & $16.3 \pm 12.6$ & -87 & .0007 & $8.2 \pm 3.7$ & $1.8 \pm 0.9$ & -78 & $<.0001$ & $3.8 \pm 3.8$ & $0.7 \pm 0.4$ & -83 & .0014 \\
\hline $\begin{array}{l}\text { First dilator } \\
\text { insertion }\end{array}$ & $333.2 \pm 167.0$ & $32.5 \pm 24.8$ & -90 & .0179 & $15 \pm 4.3$ & $2.0 \pm 1.4$ & -87 & .0003 & $4.7 \pm 1.9$ & $0.5 \pm 0.5$ & -90 & .0092 \\
\hline $\begin{array}{l}\text { Hardware } \\
\text { placement }\end{array}$ & $169.7 \pm 126.2$ & $35.1 \pm 30.7$ & -79 & $<.0001$ & $11.1 \pm 6.6$ & $2.5 \pm 1.2$ & -78 & $<.0001$ & $2.5 \pm 3.5$ & $0.4 \pm 0.3$ & -83 & .0075 \\
\hline $\begin{array}{l}\text { Hardware } \\
\text { removal }\end{array}$ & $960.0 \pm 619.6$ & $329.3 \pm 0$ & -66 & .0263 & $3.75 \pm 4.0$ & $1.5 \pm 0$ & -60 & .1150 & $1.6 \pm 2.9$ & $0.3 \pm 0$ & -84 & .1460 \\
\hline Overall & $301.5 \pm 56.3$ & $56.3 \pm 95.8$ & -81 & .0003 & $9.5 \pm 5.6$ & $2.1 \pm 1.05$ & -78 & $<.0001$ & $3.00 \pm 1.0$ & $0.50 \pm 0.2$ & -83 & $<.0001$ \\
\hline
\end{tabular}

performed with instrument navigation were compared with conventional fluoroscopy. Cumulative radiation reduction was $83 \%(P<.0001)$, reduction in $\mathrm{x}$-rays taken was $78 \%(P<.0001)$, and time reduction was $81 \%(P<.0003)$ (Table 3; Figure 2). Statistical significance held for nearly each procedure type and each surgeon studied (Table 3). The tracked tasks saved an average of 12 minutes in OR time per intervention.

Radiation reduction from use of instrument navigation ranged from $90 \%$ during dilator placement to $83 \%$ for hardware placement. The total operating time saved across all 39 averaged tasks was 123 minutes, with skin marking saving the least time at 28 minutes over 16 tasks and hardware removal saving the most time, encompassing 42 minutes of total time saved over 4 removals.

\section{DISCUSSION}

Ionizing radiation, even in small doses, can pose significant health risks to surgeons, patients, and ancillary OR staff alike. ${ }^{11-17}$ In a retrospective study by Mastrangelo et $\mathrm{al},{ }^{18}$ radiation was felt to be responsible for a 5-fold increase in cancer rates amongst orthopedic surgeons compared with nonorthopedic surgeons. A similar study by Jones et $\mathrm{al}^{19}$ showed that physicians in radiation-intensive specialties demonstrated increased rates of thyroid cancers. Alarmingly, approximately $50 \%$ of those who work in interventional suites have evidence for precataract formation. ${ }^{20}$ Given these as well as a plethora of additional studies corroborating these findings, the US Food and Drug Administration has issued a white paper to in part help express that OR radiation poses a serious health risk. ${ }^{21}$ Despite the known advantages of MISS, it is thus important to also consider its long-term adverse health effects secondary to its increased reliance on intraoperative fluoroscopy.

This radiation exposure during fluoroscopically guided spinal procedures is not insignificant. Compared with conventionally placed screws, an in vitro study by Rampersaud et $\mathrm{al}^{22}$ demonstrated that fluoroscopically assisted pedicle screw insertion resulted in a 12-fold increase in radiation exposure compared with pedicle screws inserted under direct visualization. Similarly, a prospective study by Mariscalco et $\mathrm{al}^{5}$ demonstrated that MIS lumbar discectomy resulted in up to a 20 -fold increase in radiation exposure to vital organs compared with its open counterpart. In posterior lumbar interbody fusion, not surprisingly, a $49 \%$ increase in radiation
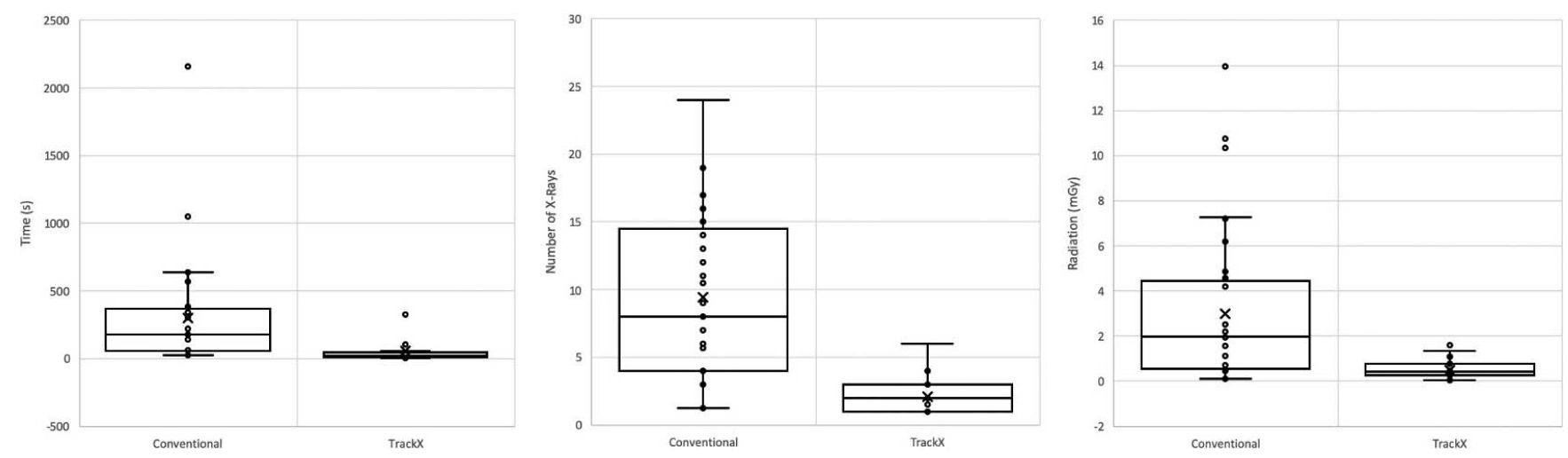

Figure 2. One-way effect analysis on overall reported time (left), number of x-rays required to perform a given portion of the procedure (middle), and radiation (right) between conventional C-arm fluoroscopy and TrackX. 
time has been found from minimally invasive techniques as well. ${ }^{23}$

As a result of these initial studies, it has become ever important to reduce radiation exposure to levels as low as reasonably achievable. Because the vast majority of radiation exposure during MISS occurs during instrumentation, reductions in radiation requirements during these portions of the procedure will stand to create the most significant change. Indeed, instrument navigational technology such as Airo CT and O-Arm were thus created with this goal in mind, and initial data on radiation reduction for these and other technologies has indeed demonstrated efficacious decreases in exposure to the surgeons and OR staff. ${ }^{24,25}$ Although these technologies minimize radiation exposure to surgeons and OR staff, they may increase radiation exposure to the patient and also result in decreased surgeon efficiency and slowed workflow. ${ }^{8}$ The high cost and space-occupying nature of these technologies has also prevented its widespread adoption across all ORs. Any instrument-navigational technology that can achieve accurate navigation without the use of preoperative or intraoperative highradiation imaging could thus lead to drastic reductions in radiation exposure to the patient, as well as OR personnel, and decrease the amount of time required to complete a given surgery. As such, we developed the novel navigational system described in this article.

The technology used in this study functions as an accessory to the C-arm fluoroscope frequently used in standard spinal procedures. Its use allows for a direct reduction in the total number of $\mathrm{x}$-rays taken during a procedure because it uses pseudolive fluoroscopy while an instrument is being tracked. The fundamental basis of the software lies in its continuous registration algorithm, where the 3dimensional reference and tool locations are updated upon each new x-ray. It allows for the system to account for any changes in patient position or anatomy throughout a procedure - a major limitation that propagates accuracy error seen in conventional navigation systems. When compared with standard fluoroscopy, initial data on this method of instrument navigation have shown upwards of $80 \%$ decreases in radiation reduction, and although entire cases have yet to be performed using this method, extrapolation of these data suggest that it uses significantly less radiation than other systems, including those that rely on preoperative or perioperative CT scans.

It should be noted that these cases were the initial cases performed by the surgeons, and therefore included their learning curve. At least in these 3 surgeons' hands, the learning curve appears to be minimal, and all 3 surgeons immediately demonstrated statistically significant decreases in operative time; such is not true of standard image guidance. ${ }^{26,27}$ Specifically, especially in the early cases, ${ }^{28}$ the learning curve can be dramatic in conventionally navigated cases. Even for experienced surgeons, time of surgery and preoperative planning time appears to be a substantial drawback to the widespread use of these technologies. ${ }^{29}$ The ability to minimize or eliminate workflow disruption and increased OR time requirements should be a major turning point to the adoption of image guidance. To the best of our knowledge, this is the first human study to show substantial time reduction with the use of a navigation system, whereas most if not all studies and technologies appear to increase preoperative planning time, intraoperative time, or both. $^{29}$

There are several important limitations to consider in this study. The first centers around the lack of controlled randomization and the inability due to technological interface to blind the proceduralist to the imaging modality being used. Given the strength of radiation reduction, it is likely that if blinding were possible, the results shown in this study would be similar. Further, this technology was only studied on a limited number of cases with only 3 attending physicians; a broader patient and physician sampling, as well as cases studied, would only help to ensure generalization of these results. Lastly, we only reviewed a limited number of tasks throughout a procedure rather than the entirety of the case. Reviewing individual tasks rather than the entirety of the procedure allowed us to internally randomize these tasks within each case, as well as effectively compare these tasks between procedures, but ultimately the whole procedure time would be of interest to study, and future work will emphasize randomizing entire procedures to TrackX or conventional fluoroscopy, or other currently used navigational systems.

Additionally, although this article describes a small number of cases, this will be the first part of a larger series of data describing the time savings and radiation savings for fluoroscopic instrument navi- 
gation. This is meant to be an initial technical safety and feasibility study that demonstrates initial proof of concept; with larger case series and data to follow. For this reason, although we cannot increase the number of cases reported for this article, the authors believe the emphasis of this study is the initial feasibility of fluoroscopic instrument navigation and its ability to assist surgeons throughout multiple procedures - with the initial data demonstrating significant time savings and radiation reduction across a multitude of common spine surgery tasks.

Lastly, this study compares fluoroscopic instrument navigation with standard $\mathrm{C}$-arm fluoroscopy. There is no true internal control. Future work will require internal controls including more commonplace CT-guided navigational systems including OArm and Airo CT. Although it would be interesting to see direct comparisons in operative time and radiation reduction between fluoroscopic instrument navigation and standard navigation, the authors chose to compare the study technology with standard fluoroscopy because standard fluoroscopy is still widely regarded as the gold standard adjuvant for spinal instrumentation. In many surgical environments, access to instrument navigational systems is costly, and in lower-volume centers, it may not be justifiable to obtain an $\mathrm{O}$ Arm or Airo CT or instrumentation robot. There is a wealth of previous literature, however, that demonstrates that even without direct comparison, the O-Arm, Airo CT, and other methods of instrument navigation currently possess a significant learning curve and significantly interrupt workflow and add time to surgery, which decreases the efficiency of a surgeon. For example, a navigational study by Ryang et $\mathrm{al}^{30}$ demonstrated that single 3-D fluoroscopic spin can add 30 minutes of OR time, and another study by Bai et $\mathrm{al}^{28}$ demonstrated increased OR times for at least 12 months after adopting one of these navigational systems. This amounts to significant cost increases, both in terms of lost OR efficiency and increased anesthesia time. In a commentary by Resnick, ${ }^{31}$ decreasing OR time by even as little as 10 minutes per case could result in significant financial savings for the hospital - thus demonstrating the significant financial effect that can be made by increases in surgeon efficiency provided by our fluoroscopic instrument navigation. The initial results of this article demonstrate that instrument navigation with $\mathrm{C}$-arm fluoroscopy can potentially make the surgeon more efficient while reducing radiation exposure and number of x-rays required to perform a given instrumentation task, thus increasing the occupational safety profile of surgery. This introduces the potential to increase surgeon efficiency and if extrapolated, has the potential to decrease hospital and OR costs.

\section{CONCLUSION}

In this small patient sample, instrument navigation caused for a reduction in OR radiation production by $83 \%$ and time by $81 \%$ for the selected tasks using instrumentation and navigation. Instrument navigation was only used on a small number of tasks throughout a given procedure, constituting for the purpose of the study just a small portion of each case. Despite that, in these 10 study procedures studied, over 2 hours of overall OR time was saved, all while requiring negligible set up time and no system calibration. To date, this is the first clinical image-guided surgery study, which suggests that image-guided technology can make a surgeon more efficient in the OR by substantively decreasing operative time.

\section{REFERENCES}

1. Iprenburg $\mathrm{M}$, Wagner $\mathrm{R}$, Godschalx A, Telfeian AE. Patient radiation exposure during transforaminal lumbar endoscopic spine surgery: a prospective study. Neurosurg Focus. 2016;40:E7.

2. Hu SS. Blood loss in adult spinal surgery. Eur Spine J. 2004;13(suppl 1):S3-S5.

3. Funao H, Ishii K, Momoshima S, et al. Surgeons' exposure to radiation in single- and multi-level minimally invasive transforaminal lumbar interbody fusion; a prospective study. PLoS One. 2014;9:e95233.

4. Mesbahi A, Rouhani A. A study on the radiation dose of the orthopaedic surgeon and staff from a mini C-arm fluoroscopy unit. Radiat Prot Dosimetry. 2008;132(1):98-101.

5. Mariscalco MW, Yamashita T, Steinmetz MP, Krishnaney AA, Lieberman IH, Mroz TE. Radiation exposure to the surgeon during open lumbar microdiscectomy and minimally invasive microdiscectomy: a prospective, controlled trial. Spine (Phila Pa 1976). 2011;36(3):255-260.

6. Costa F, Tosi G, Attuati L, et al. Radiation exposure in spine surgery using an image-guided system based on intraoperative cone-beam computed tomography: analysis of 107 consecutive cases. J Neurosurg Spine. 2016;25(5):654-659.

7. Kim CW, Lee YP, Taylor W, Oygar A, Kim WK. Use of navigation-assisted fluoroscopy to decrease radiation exposure during minimally invasive spine surgery. Spine J. 2008;8(4):584 590.

8. Nottmeier EW, Pirris SM, Edwards S, Kimes S, Bowman C, Nelson KL. Operating room radiation exposure in cone 
beam computed tomography-based, image-guided spinal surgery: clinical article. J Neurosurg Spine. 2013;19(2):226-331.

9. Hartl R, Lam KS, Wang J, Korge A, Kandziora F, Audige L. Worldwide survey on the use of navigation in spine surgery. World Neurosurg. 2013;79(1):162-172.

10. Wang TY, Hamouda F, Sankey EW, Mehta VA, Yarbrough CK, Abd-el-Barr MM. Computer-assisted instrument navigation versus conventional C-arm fluoroscopy for surgical instrumentation: accuracy, radiation time, and radiation exposure. AJR Am J Roetgenol. 2019;213(3):651-658.

11. Adams MJ, Lipshultz SE, Schwartz C, Fajardo LF, Coen V, Constine LS. Radiation-associated cardiovascular disease: manifestations and management. Semin Radiat Oncol. 2003;13(3):346-356.

12. Chou LB, Chandran S, Harris AH, Tung J, Butler LM. Increased breast cancer prevalence among female orthopedic surgeons. J Womens Health (Larchmt). 2012;21(6):683-689.

13. Freedman DM, Sigurdson A, Rao RS, et al. Risk of melanoma among radiologic technologists in the United States. Int $J$ Cancer. 2003;103(4):556-562.

14. Hayes BP, Fisher RF. Influence of a prolonged period of low-dosage $\mathrm{X}$-rays on the optic and ultrastructural appearances of cataract of the human lens. Br J Ophthalmol. 1979;63(7):457464.

15. Hoffman DA, Lonstein JE, Morin MM, Visscher W, Harris BS 3rd, Boice JD Jr. Breast cancer in women with scoliosis exposed to multiple diagnostic x rays. J Natl Cancer Inst. 1989;81(17):1307-1312.

16. Mohan AK, Hauptmann M, Freedman DM, et al. Cancer and other causes of mortality among radiologic technologists in the United States. Int J Cancer. 2003;103(2):259-267.

17. Yoshinaga S, Mabuchi K, Sigurdson AJ, Doody MM, Ron E. Cancer risks among radiologists and radiologic technologists: review of epidemiologic studies. Radiology. 2004;233(2):313-321.

18. Mastrangelo G, Fedeli U, Fadda E, Giovanazzi A, Scoizzato L, Saia B. Increased cancer risk among surgeons in an orthopaedic hospital. Occup Med (Lond). 2005;55(6):498-500.

19. Jones DP, Robertson PA, Lunt B, Jackson SA. Radiation exposure during fluoroscopically assisted pedicle screw insertion in the lumbar spine. Spine (Phila Pa 1976). 2000;25(12):1538-1541.

20. Karatasakis A, Brilakis HS, Danek BA, et al. Radiationassociated lens changes in the cardiac catheterization laboratory: results from the IC-CATARACT (Cataracts Attributed to Radiation in the CaTh lab) study. Catheter Cardiovasc Interv. 2018;91(4):647-654.

21. US Food and Drug Administration. Initiative to Reduce Unnecessary Radiation Exposure from Medical Imaging. Silver Spring, MD: US Food and Drug Administration; 2010:1-11.

22. Rampersaud YR, Foley KT, Shen AC, Williams S, Solomito M. Radiation exposure to the spine surgeon during fluoroscopically assisted pedicle screw insertion. Spine (Phila Pa 1976) 2000;25:2637-2645

23. Ntoukas V, Muller A. Minimally invasive approach versus traditional open approach for one level posterior lumbar interbody fusion. Minim Invasive Neurosurg. 2010;53(1):21-24.
24. Pennington Z, Cottrill E, Westbroek EM, et al. Evaluation of surgeon and patient radiation exposure by imaging technology in patients undergoing thoracolumbar fusion: systematic review of the literature. Spine $J$. 2019;19(8):1397-1411.

25. Riis J, Lehman RR, Perera RA, et al. A retrospective comparison of intraoperative CT and fluoroscopy evaluating radiation exposure in posterior spinal fusions for scoliosis. Patient Saf Surg. 2017;11:32.

26. Overley SC, Cho SK, Mehta AI, Arnold PM. Navigation and robotics in spinal surgery: where are we now? Neurosurgery. 2017;80(3S):S86-S99.

27. Rahmathulla G, Nottmeier EW, Pirris SM, Deen HG, Pichelmann MA. Intraoperative image-guided spinal navigation: technical pitfalls and their avoidance. Neurosurg Focus. 2014;36:E3.

28. Bai YS, Zhang Y, Chen ZQ, et al. Learning curve of computer-assisted navigation system in spine surgery. Chin Med $J$ (Engl). 2010;123:2989-2994.

29. Roser F, Tatagiba M, Maier G. Spinal robotics: current applications and future perspectives. Neurosurgery. 2013;72(suppl 1):12-18.

30. Ryang YM, Villard J, Obermuller T, et al. Learning curve of 3D fluoroscopy image-guided pedicle screw placement in the thoracolumbar spine. Spine J. 2015;15(3):467-476.

31. Resnick DK. Digital versus plain film imaging in the operating room: cost and safety considerations. Spine J. 2009;9(12):1035-1036.

Disclosures and COl: The authors, with the exception of Farah Hamouda, report no conflict of interest concerning the materials or methods used in this study or the findings specified in this paper. Ms Hamouda is a research scientist at TrackX and helped to collect and analyze the data. Ms Hamouda receives salary payment from a company involved in the manufacture of a device examined in this study. This author's role was instrumental in supplying the equipment used in this article, and her experience with the technical aspects of the imaging system were necessary to conduct this study.

Corresponding Author: Timothy Y. Wang, MD, Box 3087, Duke University Medical Center, Durham, NC 27710. Phone: (919) 423-9360; Email: timothy.wang@duke.edu.

Published 30 June 2020

This manuscript is generously published free of charge by ISASS, the International Society for the Advancement of Spine Surgery. Copyright (C) 2020 ISASS. To see more or order reprints or permissions, see http://ijssurgery.com. 